\title{
ON PAIRS OF SUBRINGS WITH A COMMON SET OF PROPER IDEALS
}

\author{
YASUYUKI HIRANO AND HISAYA TSUTSUI
}

Received 5 July 2005

A study of pairs of commutative rings with the same set of prime ideals appears in the literature. In this paper, we investigate pairs of subrings, not necessarily commutative, with a common set of proper ideals.

Throughout, all rings are associative with an identity element but not necessarily commutative. During the decade of 1980s, a series of papers appeared in the Canadian Journal of Mathematics [1, 2] that had investigated pairs of commutative rings with the same set of prime ideals. In this paper, we consider some generalizations of that study in the noncommutative setting.

Consider the ring $R=\operatorname{Hom}_{D}(V, V)$, where $V$ is a vector space over a division $\operatorname{ring} D$ with $\operatorname{dim}_{D}(V)=\aleph_{\omega_{0}}\left(\omega_{0}\right.$ is the first limit ordinal). Let $K$ be any subfield of the center of $R$, $M=\left\{f \in \operatorname{Hom}_{D}(V, V) \mid \operatorname{dim} f(V)<\aleph_{\omega_{0}}\right\}$, and $S=K+M$. Then $S$ and $R$ have the same set of countably many prime ideals. Further, $R$ and $S$ have the same set of ideals since all of their proper ideals are prime ideals. (See Blair and Tsutsui [4]). We will first observe that examples of this nature are limited in the commutative setting. It is an immediate consequence of Theorem 1 that the only possible pairs of a commutative ring $R$ and its proper unital subring $S$ with the same set of proper ideals are fields. We will then briefly investigate pairs of subrings with a common ideal. This investigation will yield the fact that a pair of rings has the same set of prime ideals if and only if they have the same set of maximal ideals. Among other things, we consider properties that pass through a pair of rings with a common set of proper ideals.

Hereafter, we reserve the term subring for a unital subring. Thus, not only a subring inherits its binary operations from its overring, but also it has the same identity element. We call subrings $R$ and $S$ of a ring (right-) ideally equal if they have the same set of proper (right) ideals, that is, $I$ is a proper (right) ideal of $R$ if and only if $I$ is a proper (right) ideal of $S$. Two rings $R$ and $S$ are called $P$ - $(M$-)ideally equal if they have the same set of prime (maximal) ideals.

Theorem 1. Suppose $S \subseteq R$ are rings which are right-ideally equal. Then either $S=R$ or $S$ and $R$ are division rings. 


\section{On pairs of subrings with a common set of proper ideals}

Proof. If $R$ and $S$ have two distinct maximal right ideals $M$ and $N$ in common, then $R=$ $M+N=S$. Thus, we may assume that $M$ is the unique maximal right ideal of $S$ and $R$. If $0 \neq a \in M$, then $a R=a S$, and for each $r \in R$ there exists $s \in S$ such that $a r=a s$, and so $r-s \in r_{R}(a)$, the right annihilator ideal of $a$ in $R$. Since $r_{R}(a)$ is a proper right ideal of $R, r_{R}(a) \subseteq S$ and so $r-s=s^{\prime}$ for some $s^{\prime} \in S$. Thus $r=s+s^{\prime} \in S$ and $R=S$. If no such nonzero $a$ exists, then $M=0$, and $S$ and $R$ are division rings.

Corollary 2. Let $R$ and $S$ be two distinct subrings of a ring. Then $R$ and $S$ are division rings if and only if $R, S$, and $R \cap S$ have the same set of proper right ideals.

Example 3. Let $K$ be a field and let $t$ be an indeterminate. Let $T=K(t)[X] /\left\langle X^{3}\right\rangle$, and set $\alpha=X+\left\langle X^{3}\right\rangle$. Then $R=K(t)\left[\alpha^{2}\right]$ is a subring of $T$, and $S=K(t-\alpha)\left[\alpha^{2}\right]$ is also a subring of $T$. Since $t \cdot \alpha^{2}=(t-\alpha) \cdot \alpha^{2}$, we have $M:=R \alpha^{2}=S \alpha^{2}=K(t) \alpha^{2}$, and it is easy to see that $M$ is the only nonzero ideal in $R$ and $S$. If $R=S$, then $\alpha \in R$, a contradiction. Hence, $R$ and $S$ are distinct subrings of the commutative ring $T$ which are ideally equal but they are not fields. Notice that if $\operatorname{char}(K)=0$, then $K \alpha^{2}$ is an ideal of $R \cap S=K+K(t) \alpha^{2}$ but it is not an ideal of $R$ nor $S$. If $\operatorname{char}(K)=p>0$, then $K\left(t^{P}\right) \alpha^{2}$ is an ideal of $R \cap S=K\left(t^{P}\right)+K(t) \alpha^{2}$ that is not an ideal of $R$ nor $S$.

We now prove two propositions on a pair of rings with a common ideal.

Proposition 4. Let $R$ and $S$ be subrings of a ring and suppose that they have a common ideal I. If $P$ is a prime ideal of $R$, then $\widetilde{P}=\{a \in S \mid I a I \subseteq P\}$ is either $S$ or a prime ideal of $S$.

Proof. Obviously $\widetilde{P}$ is an ideal of $S$. If $I \subseteq P$, then it is clear that $\widetilde{P}=S$. Suppose $I \nsubseteq P$. Then since $P$ is prime, $I \cdot 1 \cdot I=I^{2} \nsubseteq P$. Thus $\widetilde{P}$ is a proper ideal of $S$. Let $A, B$ be two ideals of $S$ such that $A B \subseteq \widetilde{P}$. Then $(I A I)(I B I) \subseteq I A B I \subseteq I \widetilde{P} I \subseteq P$. Since $P$ is prime, we have either $I A I \subseteq P$ or $I B I \subseteq P$. Hence, either $A \subseteq \widetilde{P}$ or $B \subseteq \widetilde{P}$. Therefore, $\widetilde{P}$ is a prime ideal of $S$.

By a primitive ideal $P$ of a ring $R$ we mean $R / P$ has a faithful irreducible right module.

PRoposition 5. Let $R$ and $S$ be subrings of a ring having a common ideal I. If $P$ is a primitive ideal of $R$, then $\widetilde{P}=\{a \in S \mid I a I \subseteq P\}$ is either $S$ or a primitive ideal of $S$.

Proof. Note first that since a primitive ideal is a prime ideal, $\widetilde{P}=S$ if and only if $I \subseteq$ $P$ (as was shown in the proof of Proposition 4). Suppose $I \nsubseteq P$ and let $M$ be a faithful irreducible right $R / P$-module. Then, for any nonzero $m \in M, 0 \neq m I \subseteq M$, and so $m I=$ $M$. This implies that $M$ is an irreducible right $S$-module.

We claim $M \widetilde{P}=0$. Since $M \widetilde{P}=M I \widetilde{P} \subseteq M I=M$, if $M \widetilde{P} \neq 0$ then $M \widetilde{P} I=M \neq 0$. However, $M \widetilde{P} I=M I \tilde{P} I \subseteq M P=0$, and thus $M \widetilde{P}=0$. Hence, $M$ is a right $S / \widetilde{P}$-module. Let $a$ be an element of $S$ satisfying $M a=0$. Then $M I a I=0$ and so $I a I \subseteq P$. Hence $a \in \widetilde{P}$. Therefore, $M$ is also a faithful right $S / \widetilde{P}$-module.

We denote the set of prime ideals of a ring $R$ by $\operatorname{Spec}(R)$, the set of maximal ideals of a ring $R$ by $\operatorname{Max}(R)$, and the set of primitive ideals of a ring $R$ by $\operatorname{Prim}(R)$. The following theorem shows that two rings $R$ and $S$ are $P$-ideally equal if and only if they are $M$-ideally equal. 
THeOREm 6. Let $R$ and $S$ be subrings of a ring. Then the following statements are equivalent:

(a) $\operatorname{Max}(S) \supseteq \operatorname{Max}(R)$,

(b) $\operatorname{Max}(S) \subseteq \operatorname{Max}(R)$,

(c) $\operatorname{Spec}(S)=\operatorname{Spec}(R)$,

(d) $\operatorname{Prim}(S)=\operatorname{Prim}(R)$.

Proof. Since otherwise the result is trivially true, assume that $R \neq S$. Then, if $\operatorname{Max}(S) \supseteq$ $\operatorname{Max}(R), R$ has a unique maximal ideal $M$. Let $N$ be another maximal ideal of $S$. Then since $S=M+N$, there exist $m \in M$ and $n \in N$ such that $1=m+n$. But then $n=1-$ $m \in R \backslash M$ and hence $R n R=R$. Therefore, $M^{2}=M R M=M R n R M \subseteq N$. Since $N$ is a prime ideal of $S$, this is a contradiction. Therefore $M$ is a unique maximal ideal of $S$, and hence $\operatorname{Max}(S)=\operatorname{Max}(R)=\{M\}$. This shows the equivalence of the statements (a) and (b). Suppose now that $\operatorname{Max}(S)=\operatorname{Max}(R)=\{M\}$ and let $P \neq M$ be a prime ideal of $R$. Then, by Proposition $4, \widetilde{P}=\{a \in S \mid M a M \subseteq P\}$ is a prime ideal of $S$. Since $M$ is the unique maximal ideal of $S$, we have $\widetilde{P} \subseteq M$, and so $\widetilde{P}$ is an ideal of $R$. Since $M \widetilde{P} M \subseteq P$, we obtain $\widetilde{P} \subseteq P$, and therefore $P=\widetilde{P}$ is a prime ideal of $S$. Since a primitive ideal is prime, the equivalence of the statements (a), (b), and (d) can be shown similarly by using Proposition 5.

Corollary 7. Let $R$ be a ring with unique maximal ideal $M$ and let $S=M+Z(R)$ where $Z(R)$ is the center of $R$. Then $\operatorname{Spec}(S)=\operatorname{Spec}(R)$. In this case, $M$ is also a maximal right and left ideal of $S$.

Proof. Clearly, $M$ is an ideal of $S$. Suppose that $M \subset I \subseteq S$ for a right ideal $I$ of $S$. Then, there exists $z \in I$ with $z \in Z(R) \backslash M$. Hence $R=R z R=z R$. Thus there exists $r \in R$ such that $z r=1$. But then for any $x \in R, r x=r x z r=z r x r=x r$ and therefore $r \in Z(R) \subseteq S$. This shows that $M$ is a maximal ideal and a maximal right ideal of $S$. Since $M$ is the unique maximal ideal of $R$, we have $\operatorname{Max}(R) \subseteq \operatorname{Max}(S)$, and hence, by Theorem $6, \operatorname{Spec}(S)=$ $\operatorname{Spec}(R)$.

A ring is called fully idempotent if every ideal of $R$ is idempotent. A ring $R$ is called von Neumann regular provided that for every $x \in R$ there exists $y \in R$ such that $x y x=x$. A commutative fully idempotent ring is von Neumann regular. However, the class of fully idempotent rings strictly contains the class of Von Neumann regular rings. The subring $S=x A_{1}(k)+k$ of a Weyl algebra $A_{1}(k)$ is an example of a fully idempotent ring that is not a Von Neumann regular ring.

Corollary 8. Let $R$ and $S$ be fully idempotent subrings of a ring. Then $R$ and $S$ are ideally equal if and only if $R$ and $S$ are $P$-ideally equal.

Proof. By Theorem 6, if $R$ and $S$ have the same set of prime ideals, then every ideal of $R$ and $S$ is contained in the unique maximal ideal $M$. If $I \triangleleft R$, then $I S=I(I S) \subseteq I(M S)=$ $I M \subseteq I$, and similarly, $S I \subseteq I$. Hence $I \triangleleft S$.

We are now in a position to give a few examples.

Example 9 (a pair of $P$-ideally equal rings which are not ideally equal). Let $R=\mathbb{Q}(\sqrt{3}) \oplus \mathbb{R}$ and $S=\mathbb{Q}(\sqrt{2}) \oplus \mathbb{R}$ be additive abelian groups with multiplication defined by $(a, b)(c, d)=$ 
3264 On pairs of subrings with a common set of proper ideals

$(a c, a d+b c)$. Then $R$ and $S$ are $M$-ideally equal rings with the unique maximal ideal $M=$ $0 \oplus \mathbb{R}$. Hence $R$ and $S$ are $P$-ideally equal. Let $I=0 \oplus \mathbb{Q}(\sqrt{2})$. Then $I$ is an ideal of $S$ but not of $R$.

Example 10 (a pair of rings with a nonzero common ideal that are not $P$-ideally equal). Let $K$ be a field and let $K[x]$ and $K[y]$ be two polynomial rings over $K$. Consider the ring $S=K[x] \oplus K[y]$ and its subring $R=\{(a+x f(x), a) \in S \mid a \in K, f(x) \in F[x]\}$. Then $R$ and $S$ have common ideal $I=\{(x f(x), 0) \mid f(x) \in K[x]\}$. Clearly $P=\{(0,0)\}$ is a prime ideal of $R$, but it is not a prime ideal of $S$.

Example 11 (a pair of $P$-ideally equal rings which are not fully idempotent). Let $\bar{R}$ be the ring consisting of countable matrices over $\mathbb{R}$ of the form

$$
\left[\begin{array}{cccc}
A_{m} & & & 0 \\
& a & & \\
& & a & \\
0 & & & \ddots
\end{array}\right]
$$

where $a \in \mathbb{R}$ and $A_{m}$ is an arbitrary $m \times m$ matrix over $\mathbb{R}$, and $m$ is allowed to be any positive integer. Let $\bar{S}=\bar{M}+F$ where $F$ is a subfield of the center of $\bar{R}$, and $\bar{M}$ is the subset of $\bar{R}$ consisting of all countable matrices of the form

$$
\left[\begin{array}{cccc}
A_{m} & & & \\
& 0 & & \\
& & 0 & \\
& & & \ddots
\end{array}\right],
$$

where $A_{m}$ is an arbitrary $m \times m$ matrix over $\mathbb{R}$, and $m$ is allowed to be any positive integer.

Let $S=\bar{S} \oplus \bar{M}$ and $R=\bar{R} \oplus \bar{M}$ be additive abelian groups with multiplication defined by $(a, b)(c, d)=(a c, a d+b c)$. Then, $S$ and $R$ are $M$-ideally equal rings with the unique common maximal ideal $M=\left\{\left(m_{1}, m_{2}\right) \mid m_{1}, m_{2} \in \bar{M}\right\}$ and hence, they are $P$ ideally equal. However, the ideal $I=\{(0, m) \mid m \in \bar{M}\}$ is not idempotent.

For a ring $T$, let $\mathbb{S}(T)$ be the set of all subrings $S$ of $T$ with $\operatorname{Spec}(S)=\operatorname{Spec}(T)$. Note that if $T$ has more than one maximal ideal, then $\mathbb{S}(T)=\{T\}$.

Corollary 12. Let $T$ be a ring with unique maximal ideal $M$. Then $\mathbb{S}(T)=\left\{p^{-1}(S) \mid\right.$ $S$ is a simple subring of $T / M\}$ where $p: T \rightarrow T / M$ is the canonical epimorphism.

Proof. If $X \in \mathbb{S}(T)$, then obviously $X=p^{-1}(X / M)$ and $X / M$ is a simple subring of $T / M$. Conversely, suppose that $S=X / M$ is a simple subring of $T / M$. Then $X=p^{-1}(S)$ is a subring of $T$ containing $M$ as a maximal ideal. Since $M$ is the unique maximal ideal of $T$, we have $\operatorname{Max}(T) \subseteq \operatorname{Max}\left(p^{-1}(S)\right)$. Hence by Theorem $6, p^{-1}(S) \in \mathbb{S}(T)$. 
Let $T$ be a ring with unique maximal ideal $M$. Then $\mathbb{S}(T)$ is a partially ordered set under set-theoretic inclusion. Notice that $\mathbb{S}(T / M)$ is the set of simple subrings of $T / M$, and $\varphi: \mathbb{S}(T) \rightarrow \mathbb{S}(T / M)$ defined by $\varphi\left(p^{-1}(S)\right)=S$ (where $S$ is a simple subring of $T / M$ ) gives an isomorphism between $\mathbb{S}(T)$ and $\mathbb{S}(T / M)$ by Corollary 12 . Hence, if $T / M$ is commutative, then $\mathbb{S}(T / M)$ is a complete lattice, and so is $\mathbb{S}(T)$.

Corollary 13. Let $R$ be a ring all of whose ideals are prime. Then $\mathbb{S}(R)$ forms a complete lattice.

Proof. Let $M$ be the maximal ideal of $R$ and let $Z(R)$ be the center of $R$. Let $T=M+Z(R)$. By [4, Theorems 1.3 and 4.1] of Blair and Tsutsui, $\mathbb{S}(R)=\mathbb{S}(T)$ and $T / M$ is a field. Hence $\mathbb{S}(T / M)$ is a complete lattice, and so is $\mathbb{S}(R)$.

We now investigate properties that pass through a pair of rings with a common set of proper ideals.

For a ring $R$, let $B(R)$ denote its prime radical and let $J(R)$ denote its Jacobson radical.

Proposition 14. Let $R$ and $S$ be subrings of a ring with a common ideal $I$.

(a) If $R$ is a semiprime ring and if $r_{S}(I)=\ell_{S}(I)=0$, then $S$ is a semiprime ring.

(b) If $R$ is a semiprimitive ring and if $r_{S}(I)=\ell_{S}(I)=0$, then $S$ is a semiprimitive ring.

Proof. (a) Let $P$ be a prime ideal of $R$. Then by Proposition $4, I B(S) I \subseteq I \widetilde{P} I \subseteq P$. Hence, $I B(S) I \subseteq B(R)=0$. But then since $r_{S}(I)=\ell_{S}(I)=0$, we have $B(S)=0$.

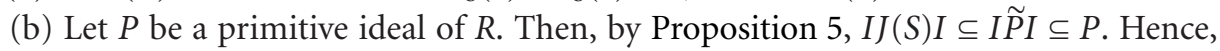
$I J(S) I \subseteq J(R)=0$. But then, since $r_{S}(I)=\ell_{S}(I)=0$, we have $J(S)=0$.

Let $R \subseteq S$ be rings with a common ideal $I$, and let $P$ be a prime ideal of $R$ with $I \nsubseteq P$. Then "lying over" holds, that is, there exists a prime ideal $Q$ in $S$ such that $Q \cap R=P$. (See, e.g., Rowen [6].)

Proposition 15. Let $R \subseteq S$ be rings with a common ideal I. If $P$ is a prime ideal of $S$ with $I \nsubseteq P$, then $P \cap R$ is a prime ideal of $R$.

Proof. Let $A, B$ be two ideals of $R$ such that $A B \subseteq P \cap R$. Then $(S A)(I S B) \subseteq S A I B \subseteq S A B \subseteq$ $S P=P$. Since $P$ is prime, $S A \subseteq P$ or $I S B \subseteq P$.

If $S A \subseteq P$, then $A \subseteq P \cap R$. Suppose that $I S B \subseteq P$. Since $I \nsubseteq P$ and since $P$ is prime, $B \subseteq S B \subseteq P$. Hence $B \subseteq P \cap R$.

Proposition 16. Let $R \subseteq S$ be rings with a common ideal I. Then if $S$ is a semiprime ring and if $I$ is an essential ideal of $S$, then $R$ is a semiprime ring.

Proof. Let $P$ is be a prime ideal of $S$. If $I \subseteq P$, then $B(R) \cap I \subseteq P$. On the other hand, if $I \nsubseteq P$, then by Proposition 15, $P \cap R$ is a prime ideal of $R$, and hence $B(R) \cap I \subseteq B(R) \subseteq$ $P \cap R \subseteq P$. Therefore, $B(R) \cap I \subseteq B(S)=0$. But then, since $R \subseteq S$ and $I$ is an essential ideal of $S$, we have $B(R)=0$.

Note that the "primitive versions" of Propositions 15 and 16 are also valid.

A fully idempotent ring is in particular, a semiprime ring.

Proposition 17. Let $R$ and $S$ be $M$-ideally equal rings with the common maximal ideal $M$. If $R$ is fully idempotent, then so is $S$, and in this case they are ideally equal. 
Proof. Let $I$ be a proper ideal of $S$. Then $I R \subseteq R I R=(R I R)^{2}=R I(R I R) \subseteq R I(R M R)=$ $R I M \subseteq R I$ and similarly, $R I \subseteq I R$. Hence $I R=R I$ is an ideal of $R$. But then $I R=(I R)^{2}=$ $I(R I R) \subseteq I M \subseteq I$ and therefore, $I$ is an ideal of $R$. This shows that $S$ is fully idempotent and the second claim now follows by Theorem 6 and Corollary 8 .

Every right ideal of a von Neumann regular is idempotent. A ring all of whose right ideals are idempotent is called a fully right idempotent ring and it has received some attention in the literature.

Proposition 18. Let $R$ and $S$ be $M$-ideally equal rings with the common maximal ideal $M$. Then if $R$ is fully right idempotent, then so is $S$, and in this case they are ideally equal.

Proof. Let $I$ be a proper right ideal of $S$ and $a \in I$. If $a \notin M$, then $S a S=S$, and hence $a \in a S=a S a S \subseteq I^{2}$. On the other hand, since $R$ is fully right idempotent, if $a \in M, a \in$ $a R=a R a R$. Now, since $R$ is in particular fully idempotent, by Proposition 17, RaR is an ideal of $S$. Since $R a R$ is the smallest ideal of $R$ containing $a$ and $S a S$ is the smallest ideal of $S$ containing $a$, we have $S a S=R a R$. Hence $a \in a R=a R a R=a S a S \subseteq I^{2}$.

The next natural question is whether or not the "regularity" passes through $M$-ideally equal rings.

Proposition 19. Let $R$ and $S$ be $M$-ideally equal rings with the common maximal ideal $M$. If $R$ is von Neumann regular, then $S$ is von Neumann regular if and only if $S / M$ is von Neumann regular.

Proof. If $R$ is von Neumann regular, then $M$ is von Neumann regular as a ring by [5, Lemma 1.3] of Goodearl. By the same lemma, if $M$ and $S / M$ are both von Neumann regular, then so is $S$.

Example 20. Let $W$ denote the $n$th Weyl algebra over a field of characteristic zero. It is well known that $W$ is a simple Noetherian domain, and hence $W$ is an Ore domain. Let $D$ denote the field of fraction of $W$. Let $R$ be the set of countable matrices over $D$ of the form

$$
\left[\begin{array}{cccc}
A_{m} & & & 0 \\
& a & & \\
& & a & \\
0 & & & \ddots
\end{array}\right]
$$

where $a \in D$ and $A_{m} \in M_{m}(D)$, the ring of $m \times m$ matrices over $D$, and $m$ is allowed to be any positive integer. Let $S$ be the same set of matrices except $a \in W$. Then $R$ and $S$ are $M$-ideally equal rings with the maximal ideal $M$ that consists of countable matrices of the form

$$
\left[\begin{array}{cccc}
A_{m} & & & 0 \\
& 0 & & \\
& & 0 & \\
0 & & & \ddots
\end{array}\right] .
$$


Any factor ring of a von Neumann regular ring is von Neumann regular, and a Noetherian von Neumann regular ring is semisimple Artinian. Therefore, $S$ is not a von Neumann regular ring since $S / M \simeq W$ is a simple Noetherian but not an Artinian ring. The fact that $R$ is a von Neumann regular ring follows immediately by the definition of a von Neumann regular ring since $M_{m}(D)$ is von Neumann regular and $0 \neq a \in D$ is invertible.

The center of a simple ring is a field and there are simple rings that are not right Noetherian. Thus, in the noncommutative setting, neither the descending nor ascending chain condition passes through a pair of ideally equal rings.

The most natural generalization of commutative rings is the class of PI-rings, that is, rings that satisfy a polynomial identity.

Proposition 21. Let $R \subset S$ be $M$-ideally equal rings with the common maximal ideal $M$. If $S$ satisfies a polynomial identity, then $R$ is right Noetherian if and only if $S$ is right Noetherian and $S / M$ is finitely generated right $R / M$-module.

Proof. Let $M$ be the maximal ideal. Since $R$ and $S$ are PI-rings, $M$ is the Jacobson radical of the two rings. Suppose that $R$ is right Noetherian. Then $M$ is a finitely generated $R$ module. Following the proof of [1, Lemma 3.27] of Anderson and Dobbs, one can show that $S$ is a finitely generated right $R$-module. $S$ is then right Noetherian and $S / M$ is a finitely generated right $R / M$-module.

Conversely, assume that $S$ is right Noetherian and $S / M$ is a finitely generated right $R / M$-module. Since $R / M$ is a simple Artinian ring, $R / M$ is a Noetherian right $R$-module. Hence, to show that $R$ is right Noetherian, it suffices to show that $M_{R}$ is Noetherian. Let $M_{1} \subseteq M_{2} \subseteq \ldots$ be an ascending chain of $R$-submodules of $M_{R}$. Then $M_{1} M \subseteq M_{2} M \subseteq \ldots$ is an ascending chain of $S$-submodules of $M_{S}$. Since $M_{S}$ is right Noetherian, there exists a positive integer $n$ such that $M_{n} M=M_{n+1} M=\ldots$. Consider next the chain $M_{n} S / M_{n} M \subseteq$ $M_{n+1} S / M_{n} M \subseteq \ldots$ This is an ascending chain of $S$-submodules of $\left(M / M_{n} M\right)_{S}$. Thus there exists a positive integer $m>n$ such that $M_{m} S / M_{n} M=M_{m+1} S / M_{n} M=\ldots$. Since $M_{m} S / M_{n} M$ is a finitely generated right $S / M$-module and since $S / M$ is a simple Artinian ring, it has finite length as a right $S / M$-module. Since $S / M$ is a finitely generated right $R / M$-module, $M_{m} S / M_{n} M$ has finite length as a right $R / M$-module. Since, for each $k>m, M_{k} / M_{n} M$ is an $R / M$-submodule of $M_{m} S / M_{n} M$, there exists a positive integer $t>m$ such that $M_{t} / M_{n} M=$ $M_{t+1} / M_{n} M=\ldots$ But then $M_{t}=M_{t+1}=\ldots$.

Example 22. Let $R=\mathbb{Q} \oplus \mathbb{R}$ and $S=\mathbb{R} \oplus \mathbb{R}$ be additive abelian groups with multiplication defined by $(a, b)(c, d)=(a c, a d+b c)$. Then $R$ and $S$ are commutative $M$-ideally equal rings with the unique maximal ideal $M=0 \oplus \mathbb{R} . M=0 \oplus \mathbb{R}$ is the only nonzero proper ideal of $S$ and hence $S$ is Noetherian. On the other hand, $0 \oplus \mathbb{Q} \subset 0 \oplus \mathbb{Q}(\sqrt{2}) \subset$ $0 \oplus \mathbb{Q}(\sqrt{2}, \sqrt{3}) \subset 0 \oplus \mathbb{Q}(\sqrt{2}, \sqrt{3}, \sqrt{5}) \subset \ldots$ is an ascending chain of ideals in $R$ that does not terminate.

\section{Acknowledgment}

We wish to thank the referee for a very helpful critique of the manuscript. 
3268 On pairs of subrings with a common set of proper ideals

\section{References}

[1] D. F. Anderson and D. E. Dobbs, Pairs of rings with the same prime ideals, Canad. J. Math. 32 (1980), no. 2, 362-384.

[2] _ Pairs of rings with the same prime ideals. II, Canad. J. Math. 40 (1988), no. 6, 13991409.

[3] F. W. Anderson and K. R. Fuller, Rings and Categories of Modules, 2nd ed., Graduate Texts in Mathematics, vol. 13, Springer, New York, 1992.

[4] W. D. Blair and H. Tsutsui, Fully prime rings, Comm. Algebra 22 (1994), no. 13, 5389-5400.

[5] K. R. Goodearl, Von Neumann Regular Rings, Monographs and Studies in Mathematics, vol. 4, Pitman, Massachusetts, 1979.

[6] L. H. Rowen, Ring Theory. Vol. I, Pure and Applied Mathematics, vol. 127, Academic Press, Massachusetts, 1988.

Yasuyuki Hirano: Department of Mathematics, Okayama University, Okayama 700-8530, Japan

E-mail address: yhirano@okayama-u.ac.jp

Hisaya Tsutsui: Department of Mathematics, Embry-Riddle Aeronautical University, Prescott, AZ 86301, USA; Department of Mathematics, Millersville University, Millersville, PA 17551, USA

E-mail address: dr.t@math-sci.net 


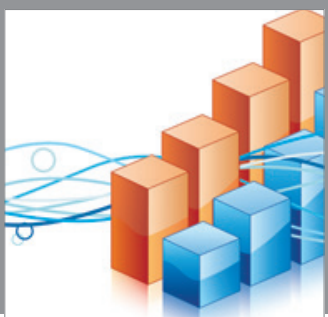

Advances in

Operations Research

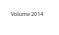

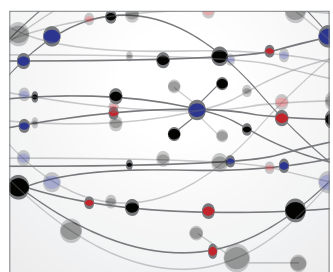

\section{The Scientific} World Journal
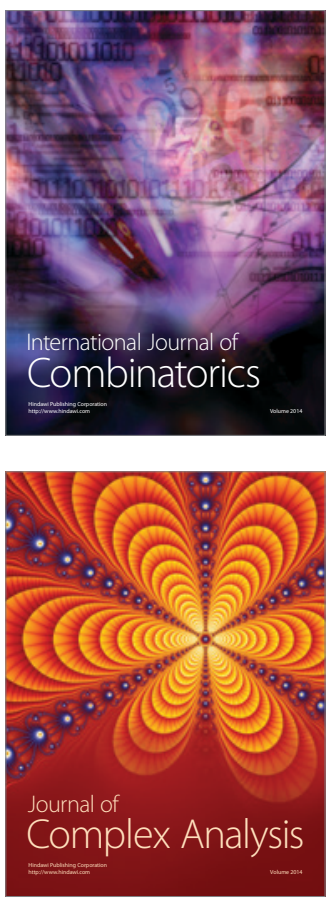

International Journal of

Mathematics and

Mathematical

Sciences
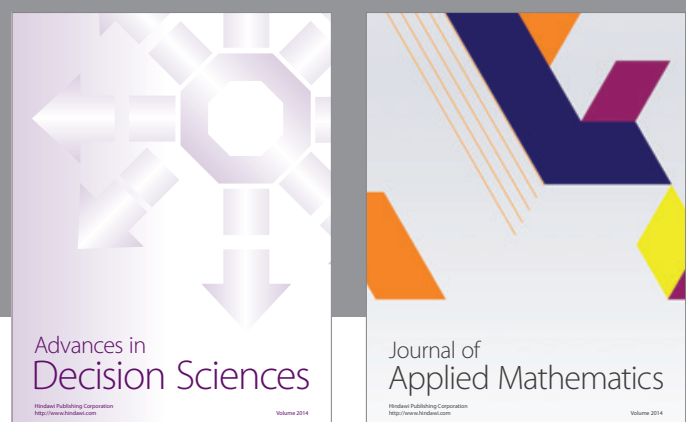

Journal of

Applied Mathematics
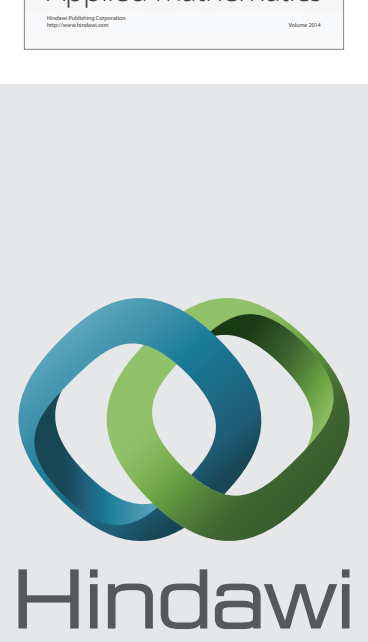

Submit your manuscripts at http://www.hindawi.com
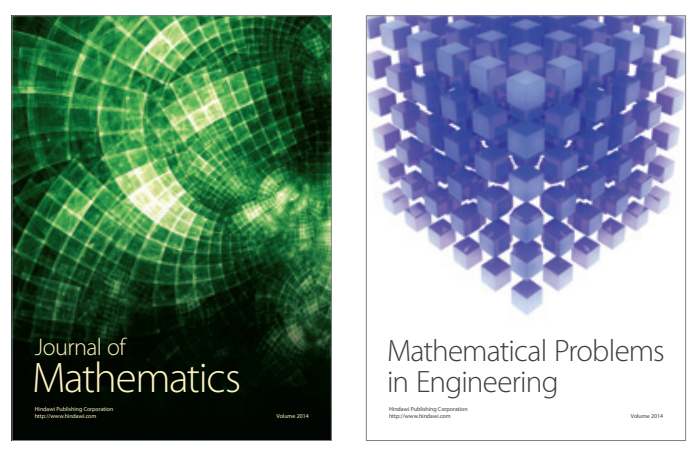

Mathematical Problems in Engineering
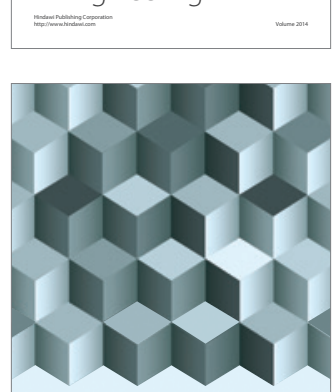

Journal of

Function Spaces
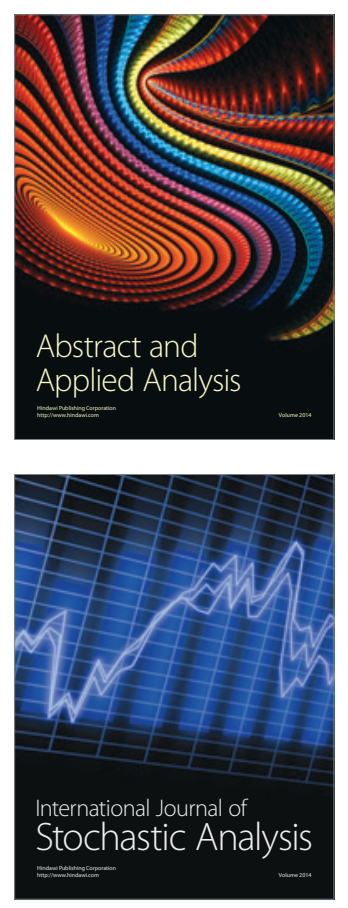

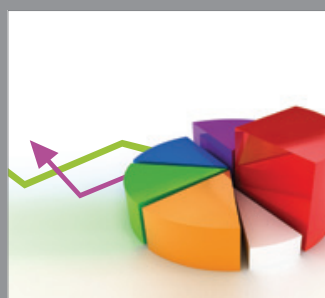

ournal of

Probability and Statistics

Promensencen
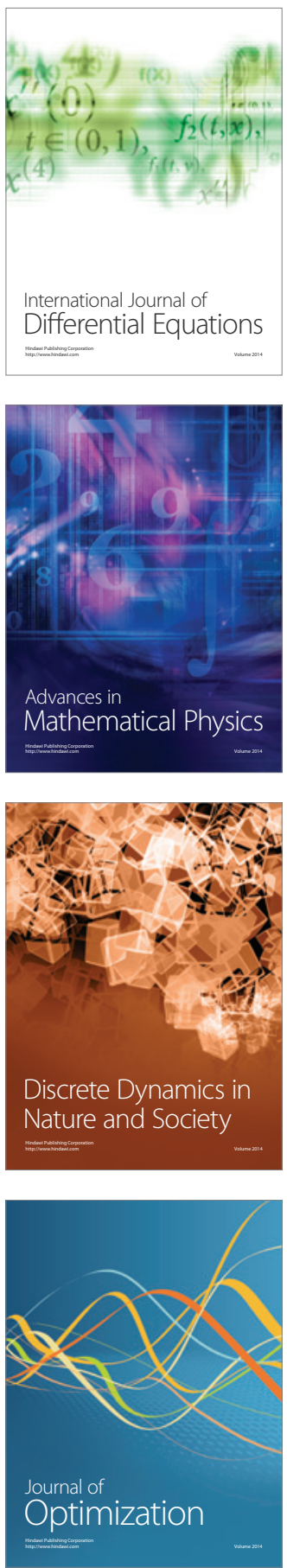